\title{
Comparative Study on Performance of Discrete Wavelength Transform and Huffman Compression Technique on 2D Signal
}

\author{
Mumtaz Anwar Hussin ${ }^{1}$, Farhana Ahmad Poad ${ }^{2 *}$, Ariffuddin Joret ${ }^{2}$ \\ ${ }^{1}$ Faculty of Electrical and Electronic Engineering, \\ Universiti Tun Hussein Onn Malaysia, Parit Raja, 86400, Batu Pahat, Johor, MALAYSIA \\ ${ }^{2}$ Department of Communication Engineering, Faculty of Electrical and Electronic Engineering, \\ Universiti Tun Hussein Onn Malaysia, Parit Raja, 86400, Batu Pahat, Johor, MALAYSIA
}

DOI: https://doi.org/10.30880/jeva.2021.02.01.002

Received 06 February 2021; Accepted 02 June 2021; Available online 30 June 2021

\begin{abstract}
Nowadays, the development of technology which involves multimedia data is widely used to help better understanding in spreading information. Image is known as 2D signal which contain huge data especially a high resolution image. This paper shows the comparison of applying lossy and lossless compression on the image data. Image compression is necessary in reducing the size of image for storage or transmission purpose to support most of the application nowadays. The technique applied in this paper is the hybrid of Discrete Wavelet Transform (DWT) technique and Huffman coding technique which are classified as lossy and lossless compression, respectively. The performance of image compression are evaluated in terms of compression ratio, Mean Square Error (MSE), Power Signal-to-Noise Ratio (PSNR), Structural Similarity Index (SSIM) and computing time. Several types of evaluation can determine better technique to apply on specific type of application. The stand-alone of each DWT and Huffman technique are evaluated before applying hybrid of DWT and Huffman technique. After conducting a comprehensive observation, the hybrid technique can compress with ratio about 1:17 to 1:27 due to the support from DWT technique that apply filter concept. The MSE value is high with the average about 69 which contributes to low PSNR value with about 29 to $30 \mathrm{~dB}$ due to the relation of PSNR equation with MSE value. Besides, the SSIM value is 0.6 or about $40 \%$ far from the original image that affect the output image. Despite of that, the computing time is fast with about 3 to 4 seconds which has been improved from stand-alone Huffman technique. Therefore, hybrid compression is capable of supporting each other techniques in stand-alone technique.
\end{abstract}

Keywords: Image compression, compression ratio, MSE, PSNR, SSIM

\section{Introduction}

Signal is data or information which can be processed with time variance to produce output data based on the input data. There are two type of signal which are analog and digital signal. Analog signal is a continuous signal in which the signal displays a smooth signal line while a digital signal is a discrete signal that is able to display the detail of each pixel value [1]. Usually, a digital signal or digital data is used to manage an analog signal due to the advantage in displaying an exact value of the data to process over the time. Therefore, digital signal is used to implement any signal processing.

\subsection{Digital Data Representation}

Basically, a digital data can be represented by bit of one ' 1 ' and zero ' 0 ' as shown in Fig. 1 or also known as binary data [2]. Generally, binary data is used to determine text data which can be referred to an ASCII code for human 
to understand on how the character is converted. Digital data is processed using an electronic device such as computer to meet the requirement based on the application's goal.

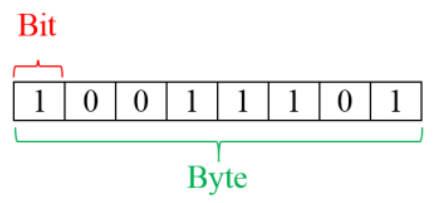

Fig. 1 - Digital data representation

An image is one of the media which are commonly used to share information between devices. It is known as a 2Dimension (2D) signal which consist the dimension detail of width and length. In addition, image processing is important in most of the application nowadays such as security system, medical imaging, wireless network [3] and etc. Some of the application required the information of output image to be $100 \%$ same with the input image to achieve the goal of application while some loss of output image data does not affect the goal of certain application.

For example, a screening test in a medical imaging application [2] need to obtain the exact data without any loss of information due to the detail of image data might risk a patient's life if there is slight information loss. Meanwhile, a social media application [1] in a wireless network system does not affect the communication between users if there are slight losses of information from the image received. Therefore, it is important to determine the purpose of the application to perform the appropriate image processing.

\subsection{Problem Statement}

Generally, an image has a large amount of data which consumes a lot of storage spaces. In wireless network system, the speed to load an image on the website is slow [4] and depends on the resolution of image. The higher the resolution of image, the slower the process to load the image. Besides, there are certain type of network does not support large data transmission to consume low power usage such as ZigBee. Therefore, compression of image is required to support the process in image transmission wirelessly. Other than that, image data shows complex character if the data is directly converted into text data which is the easiest form of data understood by human. There are certain types of compression technique that are able to encode the image data into simpler form such as Huffman coding technique. In addition, several performance on image compression technique would determine a proper technique that can be used in a certain application but most of researcher only observe the performance based on MSE and PSNR value only [5]. Hence, this paper conducted a few type of performance in applying image compression technique.

\subsection{Image Compression}

Image compression is helpful in reducing the size of image [6] or simplify the complex image data, depending on the technique of compression used. There are two types of image compression technique which are lossy and lossless compression [7]. A lossy compression is the class that would have some loss of information after applying a compression technique while a lossless compression does not have any loss of data after compression is applied. Lossy compression is suitable in most of application that emphasizes on reduction of image size to store the image or immediate transmission in wireless communication while lossless compression is suitable in the application that require the best quality of image such as medical imaging that uses a screening test to observe a very tiny cell in diagnosing the patient's health.

Since 1980's, an image file format known as Joint Photographic Expert Group (JPEG) is introduced under lossy compression technique. Until nowadays, JPEG is very useful in reducing the storage size of an image by applying a Discrete Cosine Transform (DCT) technique [8]. After that, in 1990's, lossless compression type of file format known as Portable Network Graphic (PNG) is released. PNG is very effective in reducing the size of storage while maintaining the quality of image. In this paper, the technique used are DWT technique and Huffman coding technique for lossy and lossless compression respectively. Both technique are mostly known as the technique that can produce a good performance in image compression. After that, the performances of compression are determined by evaluating the measurement of MSE, PSNR, SSIM, compression ratio and computing time.

\section{Methodology}

There are five image that are used to test compression process throughout this paper. The images are compressed stage by stage, starting with DWT technique and then followed with Huffman coding technique to observe the differences of performance between lossy and lossless compression. After that, hybrid of both DWT and Huffman technique is applied to overcome the limitation encountered in both lossy and lossless technique. 


\subsection{Discrete Wavelet Transform}

Discrete Wavelet Transform (DWT) technique is classified under lossy compression technique which resulted some loss of data after compression is made. Basically, DWT technique applied a filtering concept which divides the input image data into low frequency signal and high frequency signal. The data of high frequency is removed and only use the low frequency data [9]. This technique shows the loss of data during compression process which makes it classified in lossy compression.
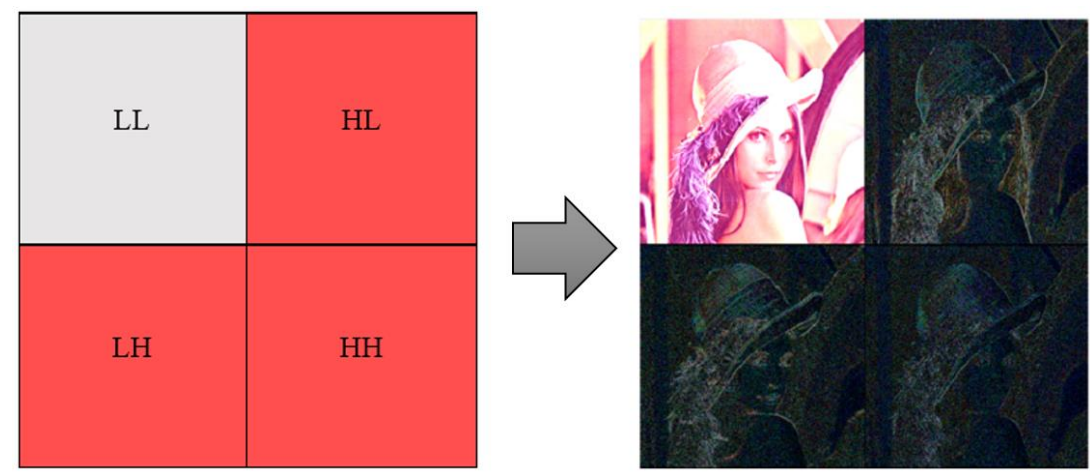

Fig. 2 - Visualization of DWT technique

Basically, image is a 2D signal which need twice of the filtering process due to the basic process of DWT is used for 1D signal. Based on Fig. 2, the input image data is divided into four parts which are approximate (LL), vertical (LH), horizontal (HL) and diagonal (HH). The only data used for decompression process is LL while other three parts are removed which cause the loss of data in DWT technique.

Based on Fig. 3, the flowchart of DWT process is shown. Firstly, the input image data is determined in which the character of image is read by the program. After that, the image is divided into Red, Green and Blue (RGB) layer [7] to undergo Forward-DWT process in each RGB layer. Next, the data is stored or transmitted in which some storage spaces is saved because the image is compressed. Next, an Inverse-DWT process is applied on each RGB layer to produce the reconstructed image. Before the image is displayed, RGB layer is regrouped to become a single coloured image.

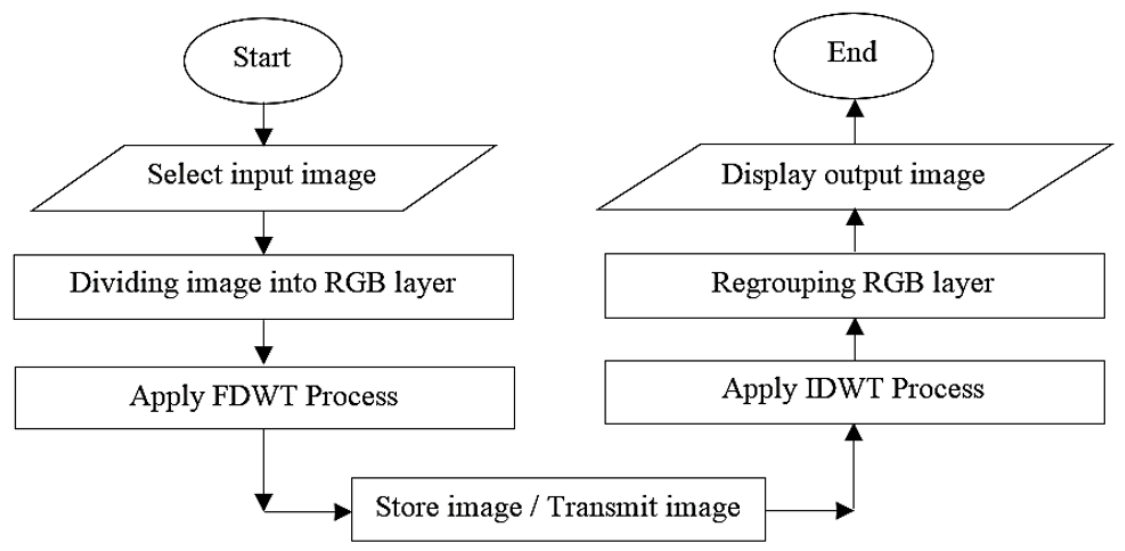

Fig. 3 - Flowchart of DWT technique

\subsection{Huffman Coding}

Huffman coding is commonly known as one of the best encoding technique which simplify the original data in efficient form and decodes the data back to become the original data [10]. Generally, Huffman coding apply a binary tree concept which develop a binary data based on the input image data. This technique is very efficient in minimizing code redundancy by using the binary data to represent the input data.

In a binary tree concept, the repeated character of input data is arranged according to the lowest probability to the highest probability of which the character is occurred as shown in Fig. 4. After that, the first two character is combined and build a binary tree data. Next, the process is repeated until the end of character. As a final point, list down the binary tree data according to the destination of each character. Lastly, the output data is arranged according to the arrangement of character from the input data. 
Afterwards, the encoded data is stored or programmed for wireless transmission. The simplified data can support most of the device to be programmed and process. An inverse process can be done to reconstruct the data. This shows that Huffman coding is helpful in maintaining the quality of data without producing any loss of information. Hence, Huffman is classified in lossless compression technique and able to achieve the goal of applications that focuses on picture quality results.

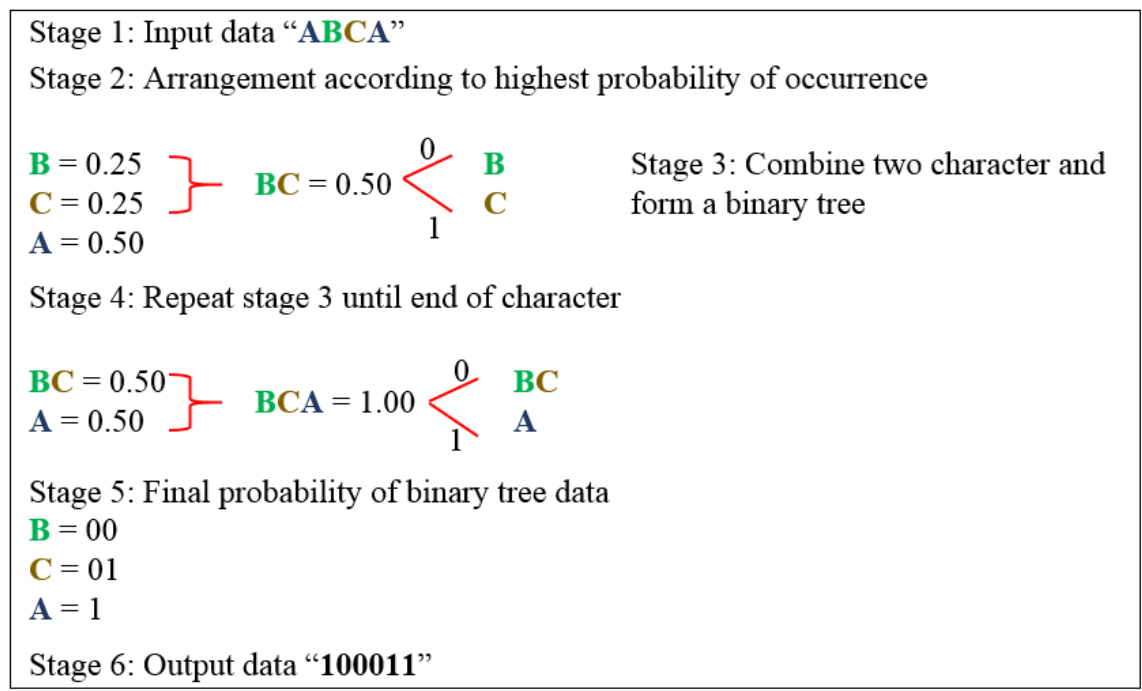

Fig. 4 - Flowchart of DWT technique

\subsection{Hybrid of DWT and Huffman Coding}

In this paper, hybrid of DWT and Huffman Coding are proposed to observe the performance of combining lossy and lossless compression technique. Based on Figure 5, the flowchart of hybrid technique shown is similar to the flowchart of DWT technique in Figure 3. The early stage starts by RGB layer division after selecting the input image. Then, lossy compression (DWT technique) is applied first to remove redundant image data at each RGB layer. After that, lossless compression (Huffman encoding technique) is applied to encode the output from DWT process and store it.

Next, the decompression process starts with Huffman decoding technique followed by inverse-DWT technique, applied to each RGB layer, received from the stage before. The output from inverse-DWT technique is regrouped to display the reconstructed image. Then, the hybrid technique is completed

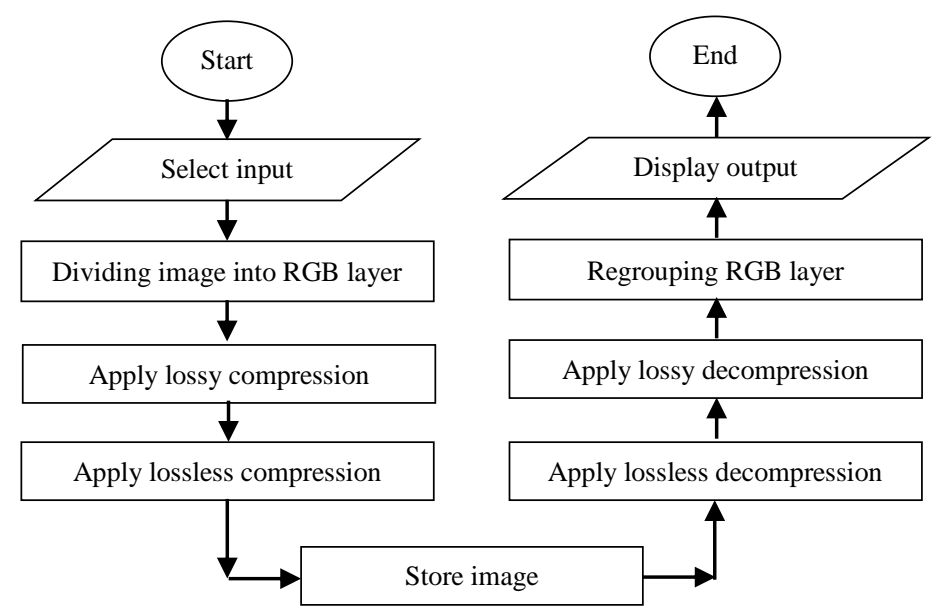

Fig. 5 - Flowchart of hybrid technique

\section{Performance Evaluation}

The performances of image compression are evaluated according to multiple terms which are Compression Ratio, Mean Square Error, Power Signal-to-Noise Ratio, Structural Similarity Index and Computing Time. 


\subsection{Compression Ratio}

Compression ratio (CR) is the ratio of original size to the compressed size as shown in equation 1 [10]. Basically, image is a large scale of matrix which consist the number of row and column. In this paper, the compression ratio is observed based on the dimension size of an image which is calculated by multiplying the row and column size of the image. In addition, a coloured image also need to multiply with three which contribute as RGB layer. Compression ratio is important to know how much is the compression can be made based on the compression technique applied.

$$
\mathrm{CR}=\frac{\text { Original image size }}{\text { Compressed image size }}
$$

\subsection{Mean Square Error}

Mean Square Error (MSE) is a measure of error produced from compression which is due to losses of data and correlation of pixel element that need to be removed. Equation 2 shows the calculation to find MSE value, where $m$ and $n$ are the total dimension size of image, $I(i, j)$ is the original image and $K(i, j)$ is the decompressed image. It determine the error according to the losses of data occur compared to the original image data [11].

$$
\operatorname{MSE}=\frac{1}{\mathrm{mn}} \sum_{i=0}^{m-1} \sum_{j=0}^{n-1}[I(i, j)-K(i, j)]^{2}
$$

$$
\text { where; } \begin{aligned}
m & =\text { row of image (in pixel) } \\
n & =\text { column of image (in pixel) } \\
K & =\text { Noisy approximation } \\
I & =\text { Noise free }(m \times n) \text { monochrome image }
\end{aligned}
$$

\subsection{Power Signal-to-Noise Ratio}

Power Signal-to-Noise Ratio (PSNR) is a common measure used for image processing to observe the quality of image. Based on equation 3, calculation of PSNR value require the MSE value [11]. The $M A X_{I}$ show at equation 4 is the maximum possible pixel value or the dynamic range of monochrome image. The result of output image shows a good quality if the PSNR value is high and the error (MSE value) is low. Hence, if the MSE value is zero, the PSNR value achieved is infinity which fulfill the characteristic of lossless compression technique.

$$
\begin{gathered}
\text { PSNR }=10 \log \frac{\left(M A X_{I}\right)^{2}}{M S E} \\
\text { for } \quad M A X_{I}=2^{b}-1=2^{8}-1=255
\end{gathered}
$$

\subsection{Structural Similarity Index}

Structural Similarity (SSIM) Index is the measurement to observe the differences between two images. In this paper, the differences between input image and output image is observed to determine the quality of image other than determining the PSNR and MSE value. Basically, the SSIM value need the information of luminance, contrast and structure of image data [11]. The maximum result of SSIM value is 1 in which the output image has exactly the same data as the input image data. Meanwhile, the result of SSIM value near to zero shows that the quality of image is poor.

\subsection{Computing Time}

Computing time is the period of time taken from the beginning of compression until decompression process is conducted. In this research, a code to count the time of compression and decompression is added which is known as 'tic' and 'toc' function which act as the sign to start counting and end counting process, respectively. The functions are applied on MATLAB programming which is the software used to program the compression technique in this paper. The location of 'tic' function is placed before compression source code and end with 'toc' after it. In the hybrid technique, the total of compression time is the time of DWT technique added with time of Huffman technique.

\section{Result and Analysis}

In this section, the characteristics of input image are elaborated and the results are analyzed according to the five performances of image compression. 


\subsection{Input Image}

In this research, there are five test images used (shown in Fig. 5) which are obtained from the SIPI, Image Database from The University of Southern California [12]. Many researchers in image processing have used the image to conduct test. In this paper, the input image has the same dimension size which is $512 \times 512$ of picture element which gives the total of 768432 pixels. The chosen image size is permanent so that other researchers are able to compare the result easily. If the larger image size is used, the compression ratio might be lower and the computing time is longer. In addition, all test image are colored and also known as high frequency image. High frequency image used to have more edge or lining design closely with each other.

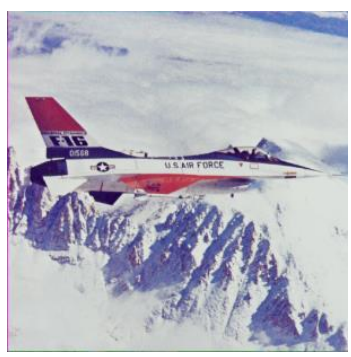

(a)

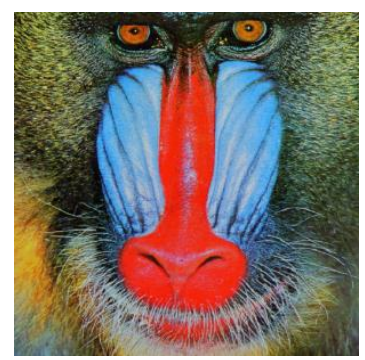

(b)

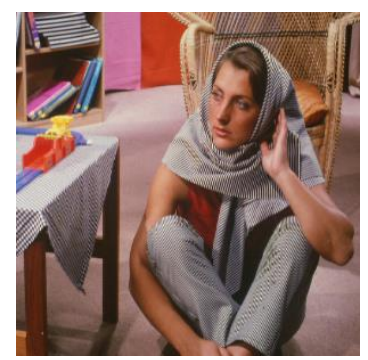

(c)

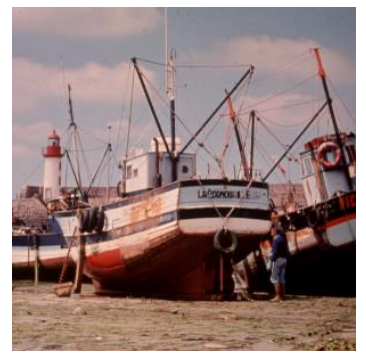

(d)

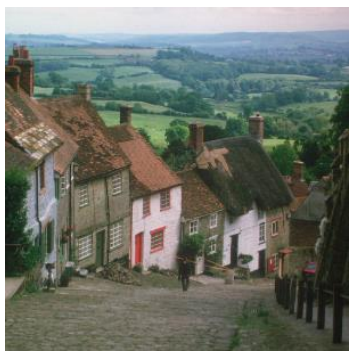

(e)

Fig. 5 - (a) Airplane; (b) Baboon; (c) Barbara; (d) Boat; (e) Gold hill [12]

\subsection{Performance of Image Compression}

The performances of DWT technique and Huffman coding technique are tabulated in Table 1 and Table 2 respectively. In DWT technique, the compression ratio is the same for all test images due to the input image has the same dimension size. The DWT technique is applied once which the reduction of image size occur only one time. The input image has the total dimension size of 768432 pixel, while the output image has 198147 pixel which resulted from $257 \times 257 \times 3$ as the data is reduced into half from the input data. Hence, the compression ratio of the original image to the compressed image is 1:3.9689. All images show the same result of compression ratio due to the same size of input image for all tested images.

Table 1 - Performance of DWT technique

\begin{tabular}{cccccc}
\hline Image & Compression Ratio & MSE & PSNR & SSIM & Computing Time \\
\hline Airplane & $1: 3.9689$ & 11.5888 & 37.5400 & 0.9546 & 0.0789 \\
Baboon & $1: 3.9689$ & 38.3016 & 32.3063 & 0.9054 & 0.0809 \\
Barbara & $1: 3.9689$ & 28.8684 & 33.4565 & 0.8740 & 0.0794 \\
Boat & $1: 3.9689$ & 15.1891 & 36.3188 & 0.9618 & 0.0832 \\
Gold hill & $1: 3.9689$ & 17.8507 & 35.8614 & 0.9243 & 0.0838 \\
\hline
\end{tabular}

Table 2 - Performance of Huffman coding technique

\begin{tabular}{cccccc}
\hline Image & Compression Ratio & MSE & PSNR & SSIM & Computing Time \\
\hline Airplane & $1: 0.1513$ & 0 & Infinity & 1 & 611.0189 \\
Baboon & $1: 0.1317$ & 0 & Infinity & 1 & 666.5590 \\
Barbara & $1: 0.1322$ & 0 & Infinity & 1 & 669.3549 \\
Boat & $1: 0.1385$ & 0 & Infinity & 1 & 636.6227 \\
Gold hill & $1: 0.1323$ & 0 & Infinity & 1 & 660.6488 \\
\hline
\end{tabular}


Consequently, Huffman encoding technique do not contribute to reducing the dimension size of image; however, it added more data after encoding process [13] which expand the dimension size of image. This is due to the length of column data is increased, while keeping the width in one row only. The extra data is stored as the dictionary or references to the coordinate of binary tree data for decompression process later. Each test image produces a slight difference of compression ratio because the RGB division is different for each image.

After that, the quality of image is evaluated based on the MSE, PSNR and SSIM value. In DWT technique, each image contributes to different value of MSE, PSNR and SSIM. Even though there is some losses of information which gives some error in the result, however the output image can still be viewed clearly. Subsequently, the PSNR value is high and SSIM value is almost 1 which contribute to good quality of output image. Plus, human naked eyes might not realize the differences between input and output image as shown in Figure 6 for the output of Barbara image due to loss of data being very low for DWT technique. Basically, DWT technique has only slight loss of data during the process in which the output image is still clear to be seen by naked eyes.

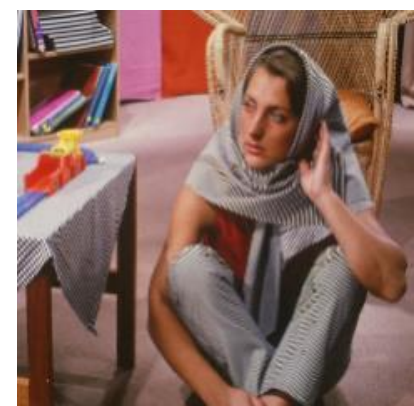

(a) Original image

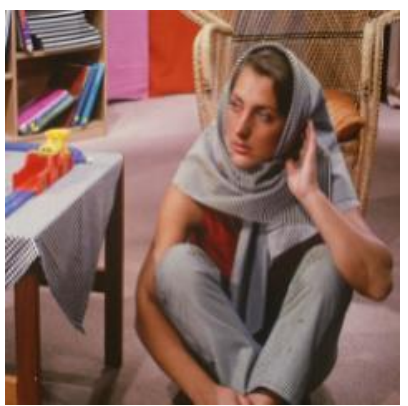

(b) Output of DWT image

Fig. 6 - Comparison of (a) original image; (b) output of DWT image

In contrast, Huffman coding technique offers the best quality of image with zero MSE value, infinity PSNR value and SSIM value of 1 which provide the output image exactly the same as the input image. This is due to Huffman coding technique which contain the dictionary data or information to rearrange the encoded data to become $100 \%$ the same as the original data after decoding process without data loss. Besides, Huffman coding technique is very useful in medical imaging application which is important in maintaining the quality of image.

Other than that, the performance of image compression can be evaluated with compression time which determines the speed of compression process. In DWT technique, all test images only used about less than one second to complete the overall process, while Huffman coding technique (shown in Table 2) used about more than 600 seconds or about 10 minutes to process. This is due to the encoding and decoding process which are required to determine the binary data according to the arrangement of frequency of occurrence from the input data and rearrange back the data in correct coordinate, respectively. Thus, DWT technique is faster to process compared to Huffman coding technique.

Table 3 - Performance of Hybrid compression technique

\begin{tabular}{cccccc}
\hline Image & Compression Ratio & MSE & PSNR & SSIM & Computing Time \\
\hline Airplane & $1: 27.4448$ & 65.3096 & 29.9819 & 0.6222 & 3.3058 \\
Baboon & $1: 22.5856$ & 80.0924 & 29.0955 & 0.6075 & 4.1615 \\
Barbara & $1: 17.9285$ & 71.6548 & 29.5880 & 0.6442 & 4.0408 \\
Boat & $1: 24.4127$ & 65.5028 & 29.9735 & 0.6752 & 3.6385 \\
Gold hill & $1: 22.6448$ & 64.5641 & 30.0325 & 0.6119 & 4.0545 \\
\hline
\end{tabular}

In the last stage, hybrid of DWT and Huffman is applied on the five images. Based on the performance of hybrid result in Table 3, hybrid technique can compress about 27 to 17 ratio to the original image. The images are able to have high compression due to the ability of DWT technique which reduces three times from the original image size [14]. The amount of compression are different for each image due to the image pixel value in each RGB layer being different in each image. Huffman encodes the data based on the probability of occurrence of the pixel value to build the binary tree. Hence, the arrangement of binary output are different in each image which effect the total dimension size of compressed image.

The overall MSE value is about 64 to 80 which considered as high data loss. Meanwhile, the overall PSNR value is about 29 to $30 \mathrm{~dB}$, and SSIM value is about 0.6 for all tested images. The measurement are attributed to the quality of output image as shown in Table 4. The output image shows a bit blurry but still able to display similar to the original image. The quality of images has dropped due to the high compression result by hybrid of both DWT and Huffman technique. Besides, the different of total amount of data loss are different in each image due to the characteristic of image whether it contain higher frequency data or lower frequency data as mentioned in Section 4.1. Baboon and 
Barbara image has higher frequency data while Airplane, Boat and Gold hill image has lower frequency data. Hence, the amount of data loss is higher for Baboon and Barbara image.

Table 4 - Comparison of original image with hybrid compression image

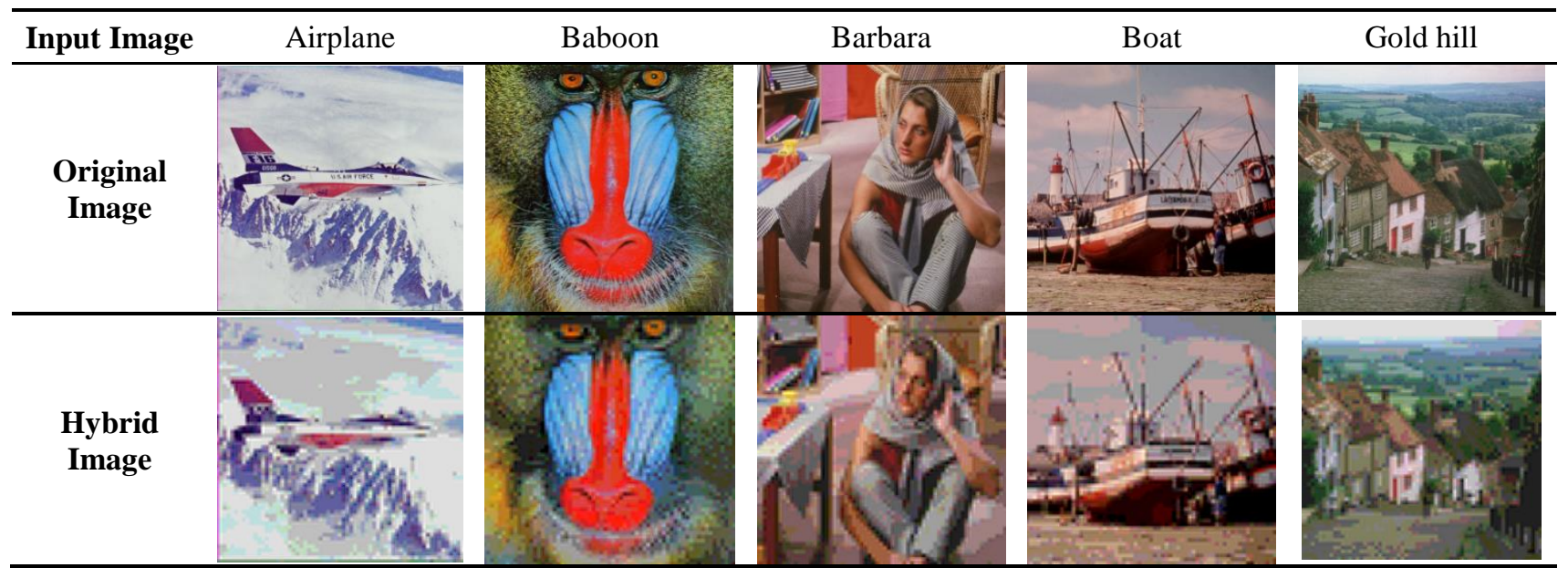

Other than that, the computing time for hybrid technique only took about 3 to 4 seconds which is fast computing time where it need to process both of lossy and lossless compression. The amount of time has been greatly reduced compared to stand-alone Huffman technique with about $92 \%$. Hence, the combination of DWT and Huffman coding techniques is able to shorten the computing time. Based on the result, higher frequency data (Baboon and Barbara image) has about 0.4 to 0.9 second higher computing time than lower frequency image. Therefore, hybrid compression technique can be applied to support the application that focuses on compression ratio and computing time such as wireless communication or security system to detect robbery. For example, when a robber is detected by any of the moving sensor in a house, the security camera automatically capture the image and it is sent to the owner's smart phone wirelessly, so the compression ratio and computing time are more important than having a high quality of output image. Plus, the owner can immediately call the police station for fast action and capture of the robber.

A very similar compression technique is done by H. Singh and S. Rana [15] which also applying hybrid of lossy compression using Discrete Cosine Transform (DCT) and DWT technique and lossless compression using Huffman technique. The author only evaluated the performance in terms of MSE, PSNR and computing time with result in 169.56, 25.87dB and 31.73 seconds, respectively. The result shows high amount of error with poor image quality conducted on Lena image which has total dimension size of 786432 pixel, the same as the total dimension size of input image test in this paper. In addition, the computing time of their hybrid technique exceeds half a minute which is quite a long time compared to the proposed hybrid technique in this paper. The author does not state the specific reason of their result; however, it might due to the flow of compression where the author only apply compression on each RGB layer during the DWT stage only.

\section{Conclusion}

In the first stage, DWT technique is able to provide some reduction of image size. It can be processed within short amount of time and the output image produced is good which only shows a less difference between the original images according to the measurement of MSE, PSNR and SSIM. In the second stage, Huffman coding technique is unable to reduce the image size and it uses an enormous amount of time to complete the process. However, the quality of image produced by Huffman coding technique is outstanding due to no loss of image data along the process. In the last stage, hybrid of DWT and Huffman technique is applied and observed. Hybrid technique can reduce the image size which makes the compression ratio gain. The quality of image in hybrid technique shows low quality especially on higher frequency image which contain more edge and lining design. Meanwhile, the computing time is fast which took about less than 4.1 second to complete both of DWT and Huffman process. Therefore, this paper is able to show the performance of 2D signal or image compression by using lossy compression (DWT technique) and lossless compression (Huffman coding technique). Furthermore, the hybrid of both lossy and lossless compression is also conducted and evaluated according to the measurement of compression ratio, MSE, PSNR, SSIM and computing time.

\section{Acknowledgement}

A special thanks to Research Management Centre (RMC), Universiti Tun Hussein Onn Malaysia (H180 Tier 1 Fund) and Registrar Office for sponsoring the research and development of this project. 


\section{References}

[1] V. Tyagi, "Understanding Digital Image Processing," Underst. Digit. Image Process., 2018

[2] A. J. Hussain, A. Al-Fayadh, and N. Radi, Image Compression Techniques: A Survey in Lossless and Lossy Algorithms, vol. 300, no. March. Elsevier B.V., 2018

[3] S. A. Aliesawi, D. S. Alani, and A. M. Awad, "Secure image transmission over wireless network," Int. J. Eng. Technol., vol. 7, no. 4, p. 2758, 2018

[4] A. Ali, Y. Ming, S. Chakraborty, and S. Iram, "A Comprehensive Survey on Real-Time Applications of WSN," Futur. Internet, pp. 1-22, 2017

[5] A. V. Dvorkovich, N. B. Novinsky, and D. N. Diep, "New Approach to Improving the Quality for Images Processed with Fractal Compression," 2020 22th Int. Conf. Digit. Signal Process. its Appl. DSPA 2020, vol. 10, pp. 23-26, 2020

[6] M. A. Rahman, M. Hamada, and J. Shin, "The Impact of State-of-the-Art Techniques for Lossless Still Image Compression," Electron. 2021, vol. 10, pp. 1-40, 2021

[7] A. J. Qasim, R. Din, and F. Q. A. Alyousuf, "Review on Techniques and File Formats of Image Compression," Bull. Electr. Eng. Informatics, vol. 9, no. 2, pp. 602-610, 2020

[8] D. Chand, B. Singh, and H. Kumar, "Study of JPEG Compression with Different DCT Methods," no. March, 2019

[9] M. A. Hussin, F. A. Poad, and A. Joret, "A Comparative Study on Improvement of Image Compression Method using Hybrid DCT-DWT Techniques with Huffman Encoding for Wireless Sensor Network Application," Int. J. Integr. Eng., vol. 11, no. 3, pp. 149-158, 2019

[10] Y. A. Salih, A. Ali Mohammed, and L. Edwar George, "Improved Image Compression Scheme Using Hybrid Encoding Algorithm," Kurdistan J. Appl. Res., vol. 4, no. 2, pp. 90-101, 2019

[11] U. Sara, M. Akter, and M. S. Uddin, "Image Quality Assessment through FSIM, SSIM, MSE and PSNR-A Comparative Study," J. Comput. Commun., vol. 07, no. 03, pp. 8-18, 2019

[12] A. G. Weber, "The USC-SIPI Image Database : Version 6," University of Southern California, Los Angeles, U.S.A, 2018

[13] R. Starosolski, "Hybrid Adaptive Lossless Image Compression Based on Discrete Wavelet Transform," Entropy, vol. 22, no. 7, 2020

[14] M. A. Hussin, F. A. Poad, and A. Joret, "A Comparative Study On Image Compression Method Using StandAlone DWT Techniques and Hybrid of DWT with Huffman Coding Technique for WSN Application," Int. J. Adv. Trends Comput. Sci. Eng., vol. 9, no. 1.4 Special Issue, pp. 117-123, 2020

[15] H. Singh and S. Rana, "Image Compression Hybrid using DCT , DWT , Huffman," Int. J. Sci. Eng. Res., vol. 3, no. 8, pp. 1-4, 2012 